"Supporting information"

\title{
A suitable combination of electrodes for simultaneous reduction of nitrates and oxidation of ammonium ions in an explosive industry wastewater
}

Rohit Chauhan, Vimal Chandra Srivastava*

Department of Chemical Engineering, Indian Institute of Technology Roorkee, Roorkee, Uttarakhand, 247667, India

*Corresponding author: Tel.: +91-1332-285889; Fax: +91-1332-276535. E-mails: vimalcsr@yahoo.co.in, vimalfch@iitr.ac.in (VCS), rohitchn18@ gmail.com (RC).

Table S1. $1^{\text {st }}$ and $\mathrm{n}^{\text {th }}$ order kinetic analysis for the electrochemical denitrification of the actual wastewater at different values of $\mathrm{j}$ and wastewater $\mathrm{pH}$.

\begin{tabular}{|c|c|c|c|c|}
\hline $\mathbf{j}\left(\mathbf{A ~ \mathbf { m } ^ { - 2 } )}\right.$ & Rate constant $(k)$ & Order of reaction $(n)$ & $\mathbf{R}^{\mathbf{2}}$ & ARE (\%) \\
\hline \multicolumn{5}{|c|}{ Effect of current density $(\mathbf{j})$ at wastewater natural $\mathbf{p H}$} \\
\hline \multicolumn{5}{|c|}{ For first order reaction rate, $\mathrm{k}_{1}=\mathrm{min}^{-1}$} \\
\hline 35.71 & $2.4 \times 10^{-3}$ & 1 & 0.81 & 3.6 \\
\hline 71.43 & $2.7 \times 10^{-3}$ & 1 & 0.80 & 4.4 \\
\hline 107.14 & $2.7 \times 10^{-3}$ & 1 & 0.70 & 5.2 \\
\hline 142.86 & $2.9 \times 10^{-3}$ & 1 & 0.72 & 5.5 \\
\hline \multicolumn{5}{|c|}{ For $\mathrm{n}^{\text {th }}$ order reaction rate, $\mathrm{k}_{\mathrm{n}}=\left(\mathrm{mg} \mathrm{L}^{-1}\right)^{(1-\mathrm{n})} \min ^{-1}$} \\
\hline 35.71 & $3.3 \times 10^{-5}$ & 2.3 & 0.88 & 7.6 \\
\hline 71.43 & $4.1 \times 10^{-5}$ & 2.3 & 0.87 & 9.2 \\
\hline 107.14 & $5.0 \times 10^{-5}$ & 2.3 & 0.82 & 12.3 \\
\hline 142.86 & $5.8 \times 10^{-5}$ & 2.3 & 0.83 & 13.1 \\
\hline \multicolumn{5}{|c|}{ Effect of wastewater $\mathrm{pH}$ and $\mathbf{j}=107.14 \mathrm{~A} \mathrm{~m}^{-2}$} \\
\hline \multicolumn{5}{|c|}{ For first order reaction rate, $\mathrm{k}_{1}=\min ^{-1}$} \\
\hline 3 & $2.6 \times 10^{-3}$ & 1 & 0.88 & 4.3 \\
\hline 6 & $3.0 \times 10^{-3}$ & 1 & 0.90 & 4.0 \\
\hline 9 & $3.1 \times 10^{-3}$ & 1 & 0.90 & 3.8 \\
\hline 12 & $3.4 \times 10^{-3}$ & 1 & 0.90 & 4.3 \\
\hline \multicolumn{5}{|c|}{ For $\mathrm{n}^{\text {th }}$ order reaction rate, $\mathrm{k}_{\mathrm{n}}=\left(\mathrm{mg} \mathrm{L}^{-1}\right)^{(1-\mathrm{n})} \min ^{-1}$} \\
\hline 3 & $1.7 \times 10^{-5}$ & 2.4 & 0.95 & 3.3 \\
\hline 6 & $2.1 \times 10^{-5}$ & 2.4 & 0.97 & 3.6 \\
\hline 9 & $2.3 \times 10^{-5}$ & 2.4 & 0.97 & 4.1 \\
\hline 12 & $2.7 \times 10^{-5}$ & 2.4 & 0.97 & 4.4 \\
\hline
\end{tabular}




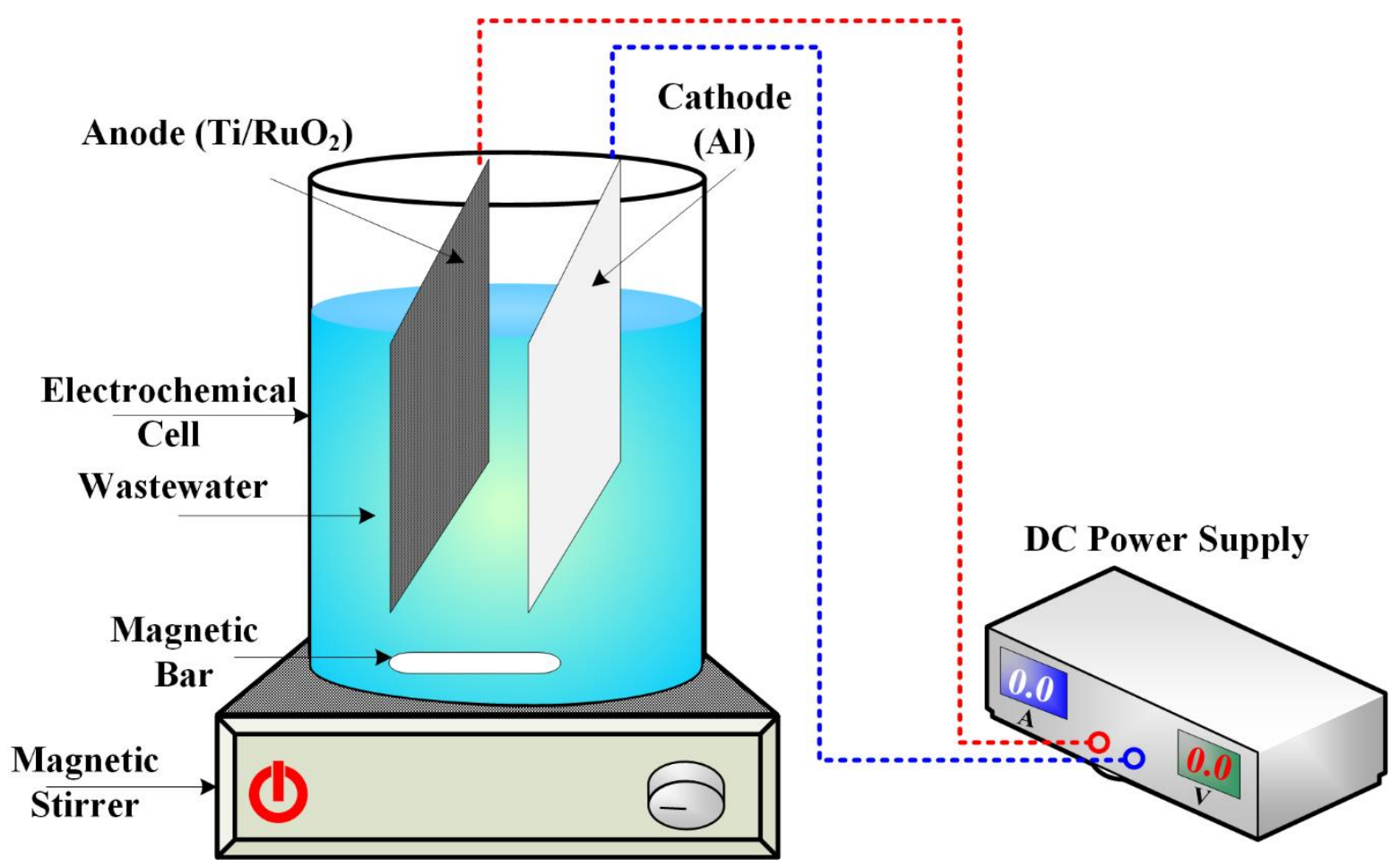

Fig. S1. Schematic diagram of the electrochemical batch denitrification of the actual wastewater. 


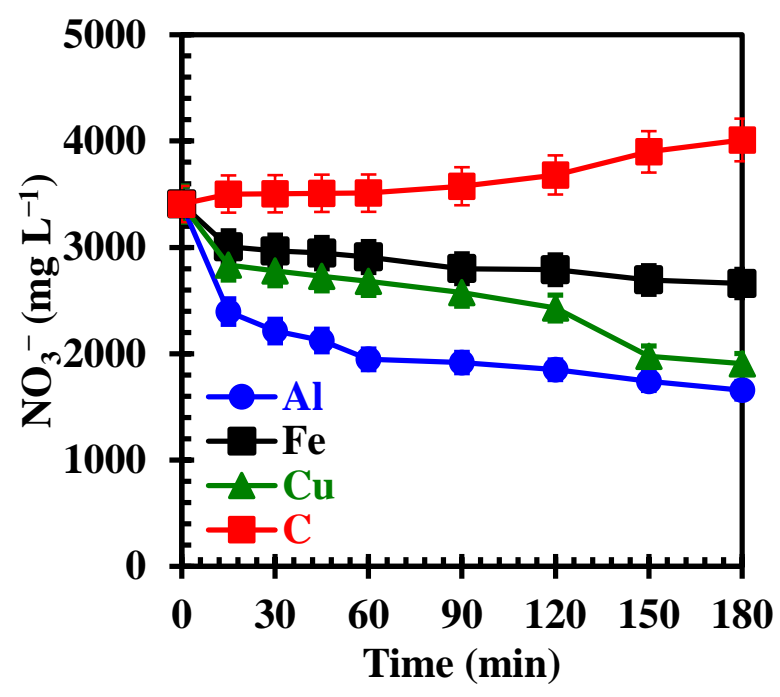

(a)

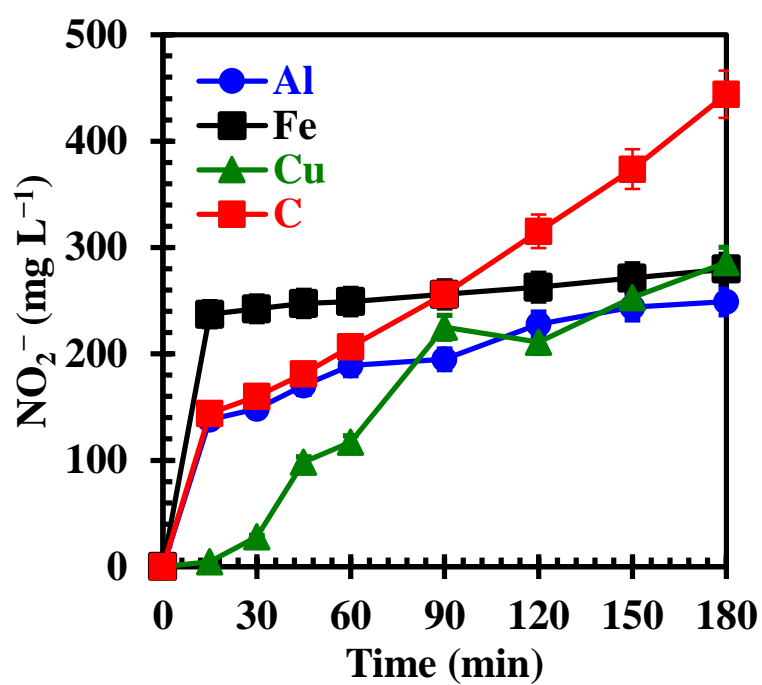

(b)

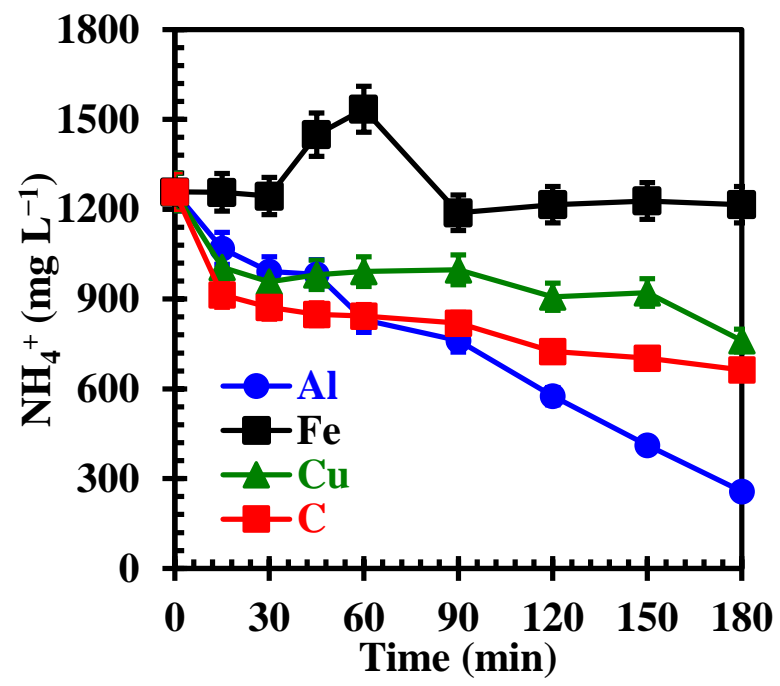

(c)

Fig. S2. Effect of different cathode material, i.e., $\mathrm{Al}, \mathrm{Fe}, \mathrm{Cu}$, and $\mathrm{C}$ on the (a) $\mathrm{NO}_{3}{ }^{-}$reduction; (b) $\mathrm{NO}_{2}{ }^{-}$ion generation; (c) $\mathrm{NH}_{4}{ }^{+}$ion distribution for electrochemical treatment of wastewater at $\mathrm{j}=142.86 \mathrm{~A} \mathrm{~m}^{-2}$. 


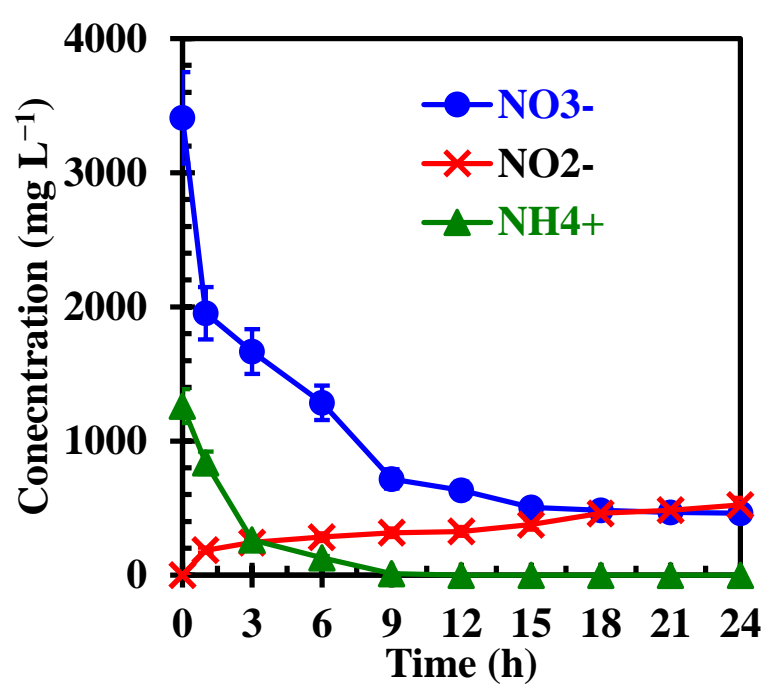

Fig. S3. Effect of longer processing time of $24 \mathrm{~h}$ on the $\mathrm{NO}_{3}{ }^{-}$reduction, $\mathrm{NO}_{2}{ }^{-}$ion generation, and $\mathrm{NH}_{4}{ }^{+}$ion distribution for electrochemical treatment of wastewater by using $\mathrm{Al}$ cathode and $\mathrm{Ti} / \mathrm{RuO}_{2}$ anode at $\mathrm{j}=142.86 \mathrm{~A} \mathrm{~m}^{-2}$. 